\title{
Modified Docetaxel, Cisplatin, and Fluorouracil (mDCF) as a Neoadjuvant Chemotherapy for Non-metastatic Esophageal Cancer (nMEC)
}

\author{
Adedayo A. Onitilo, MD, PhD, MSCR, FACP; Trista J. Stankowski-Drengler, MD, MS; \\ Oyewale Shiyanbola, MBBS, MPH, PhD; Jessica Engel, DNP, MS; Sabo Tanimu, MD; \\ Seth O. Fagbemi, MD; and Ya-Huei Li, PhD
}

\begin{abstract}
Objective: Perioperative chemotherapy can potentially downstage esophageal cancer, reducing the risk of early systemic dissemination. One recommended neoadjuvant regimen for managing gastroesophageal junction and esophageal cancer is docetaxel, cisplatin, and 5-fluorouracil (DCF). To address the high toxicity profile of DCF, modifications in dosages and treatment intervals have been studied. We integrated a modified DCF regimen $(\mathrm{mDCF})$ into a multimodal treatment approach for non-metastatic esophageal cancer (nMEC). Retrospectively, we sought to describe our community experience of administrating neoadjuvant $\mathrm{mDCF}$ to patients with nMEC.
\end{abstract}

Design: Patients diagnosed with nMEC between August 2008 and November 2017 and prescribed mDCF were identified for retrospective review. Outcomes of interest included disease-free survival (DFS), overall survival (OS), and hematologic toxicities. Analyses were performed using SAS 9.4.

Results: Thirty patients met inclusion criteria with a median age of 64.9 years; $90 \%$ were male. The 2 -year and 5-year DFS was $60.8 \%$ and $41.7 \%$, respectively, for adenocarcinoma and $71.4 \%$ and $71.4 \%$ for squamous cell carcinoma (SCC). The 2 -year and 5 -year OS was $64.9 \%$ and $44.5 \%$, respectively, for adenocarcinoma and $71.4 \%$ and $71.4 \%$ for SCC. Both DFS and OS decreased with increasing disease stage, histology (adenocarcinoma versus squamous), esophageal compared to esophagogastric-junction involvement, and without surgical intervention. Frequent toxicity grades for leukopenia and thrombocytopenia were Grades I and II.

Conclusion: Using an mDCF regimen in combination with chemoradiation $+/$ - surgical resection in a community setting appears to have an acceptable toxicity profile as well as DFS and OS outcomes compared to chemotherapeutic regimens reported in other similar studies.

Keywords: Modified docetaxel, cisplatin, and fluorouracil; Non-metastatic esophageal cancer; Disease-free survival; Overall survival; Hematologic toxicities; Community

Corresponding Author: Adedayo A. Onitilo, MD, PhD, MSCR, FACP, Marshfield Clinic Health System - Weston Center, 350I Cranberry Blvd., Weston, WI 54476 USA, Tel: (7I5) 393-1407, Fax: (7I5) 393-I399, Email: onitilo.adedayo@ marshfieldclinic.org, ORCID ID: https://orcid.org/0000-000I-9/85-0606
Received: April 20, 2020

Revised: November 30, 2020

Accepted: January 4, 2021

doi: $10.3121 / \mathrm{cmr} .2021 .1573$ 
$\mathrm{W}$ ith the lowest 5-year relative survival (19\%) among all cancer types, esophageal cancer is a fatal malignancy with an estimated 16,080 deaths in the United States (US) in 2019. ${ }^{1}$ Although it represents only $1 \%$ of all newly diagnosed cancers, esophageal cancer accounts for about $3 \%$ of all cancer deaths in the US. ${ }^{2,3}$ In addition to other histologic types, squamous cell carcinoma and adenocarcinoma are the most frequently diagnosed variants of esophageal cancer, representing $31.3 \%$ and $64.1 \%$ of all esophagus carcinoma, respectively. ${ }^{3,4}$ Although race/ethnicity plays a role in the survival of esophageal cancer, patients with esophageal squamous cell carcinoma (SCC) tend to have poorer survival rates than patients with esophageal adenocarcinoma (AC). ${ }^{5,6}$

Surgery plays an essential role in controlling local/regional esophageal carcinoma and optimizing treatment outcomes; however, it has a limited impact on survival for those with advanced esophageal carcinoma. ${ }^{7-10} \mathrm{~A}$ multimodal treatment approach combining surgical and non-surgical therapies personalized to the patient's needs, disease type, and stage, has been recommended by the European Society for Medical Oncology Guidelines Committee in the management of esophageal carcinoma. ${ }^{9-11}$ Perioperative chemotherapy has the potential advantage of downstaging esophageal cancer and reducing the risk of early systemic dissemination. ${ }^{9-11}$ Indeed, the survival advantage of perioperative neoadjuvant strategy over surgery alone in SCC and AC was confirmed in multiple metaanalyses. ${ }^{12-14}$ Still, the optimal neoadjuvant regimen has not been well identified.

In the last few decades, the first line treatment for esophageal cancerhas consisted of two to threeneoadjuvant chemotherapeutic agents with or without concurrent radiotherapy. ${ }^{12-14}$ The most frequently seen combination of neoadjuvant chemotherapy has been the combination of cisplatin and 5-fluorouracil (CF). However, the dosage, cycle, and interval of administration of the CF regimen has varied in clinical trials. ${ }^{12-14}$ The combination of docetaxel, cisplatin, and 5-fluorouracil (DCF) are frequently utilized in advanced esophageal SCC and AC. Ferri and colleagues ${ }^{15}$ conducted a phase II multicenter trial studying the effectiveness and toxicity of perioperative DCF (three 3-week cycles DCF [docetaxel $75 \mathrm{mg} / \mathrm{m}^{2}$ on day 1 , cisplatin $75 \mathrm{mg} / \mathrm{m}^{2}$ on day 1, 5-FU $750 \mathrm{mg} / \mathrm{m}^{2}$ for 120 hours] before and after surgery) for locally advanced esophageal and gastric adenocarcinoma. The findings of their study supported that not only is the DCF regimen the first line treatment for advanced gastric adenocarcinoma, but it is also a tolerable and effective neoadjuvant regimen for the management of gastroesophageal junction and esophageal cancer. ${ }^{15,16}$ Additionally, two 3-week DCF cycles have shown great effectiveness in managing advanced, metastatic, or recurrent esophageal squamous cell carcinoma when concurrently administered with radiotherapy. ${ }^{17,18}$ However, a major concern related to DCF was raised due to the significant severe adverse events (Grade III/IV adverse event of $70 \%$ ), especially neutropenia (about $80 \%$ ). ${ }^{16,17}$
Due to the relatively high toxicity profile of DCF, some clinicians have explored the option of modifying the dosage and/or frequency of this chemotherapy regimen with the hopes of maintaining effectiveness while decreasing the toxicity. A modified weekly DCF (docetaxel $35 \mathrm{mg} / \mathrm{m}^{2}$ and cisplatin 25 $\mathrm{mg} / \mathrm{m}^{2}$ on days $1,8,15,29,36,43,50$, and $57 ; 5-\mathrm{FU} 180 \mathrm{mg} / \mathrm{m}^{2}$ on days $1-21$ and $5-\mathrm{FU} 150 \mathrm{mg} / \mathrm{m}^{2}$ on days 29-63) added to 50 Gy radiotherapy described by Pasini and colleagues ${ }^{19,20}$ showed a better pathological complete remission and lower rates of severe adverse events (Grade III/IV neutropenia: 13.5\%; Grade III/IV nonhematological toxicity: $32.4 \%$ ). Shah and colleagues ${ }^{21}$ have also described another modified DCF (mDCF) regimen (docetaxel $40 \mathrm{mg} / \mathrm{m}^{2}$ on day 1 , cisplatin $40 \mathrm{mg} / \mathrm{m}^{2}$ on day 3 , 5 -FU $2000 \mathrm{mg} / \mathrm{m}^{2}$ on days $1-2$, leucovorin $400 \mathrm{mg} / \mathrm{m}^{2}$ on day 1 ) provided every 2 weeks with/without bevacizumab, which was found to extend survival with a lower toxicity profile (Grade III/ IV neutropenia $50-60 \%$ ) in contrast to two 3-week DCF cycles in the management of metastatic gastroesophageal adenocarcinoma. ${ }^{21,22}$ Our institution also adopted an $\mathrm{mDCF}$ regimen and integrated its use into our multimodal treatment approach for patients with non-metastatic esophageal cancer (nMEC). Our mDCF regimen was similar to that described by Shah et $\mathrm{al}^{21,22}$ with two important differences: (1) cisplatin is administrated on day 1 in our regimen but not on day 2 or day 3 as presented in Shah's trials; and (2) leucovorin and granulocyte colony-stimulating factors are provided. With a decade of experience utilizing the $\mathrm{mDCF}$ regimen in conjunction with radiation $+/$ - surgery for patients with $\mathrm{nMEC}$, we sought to describe our experience of administrating neoadjuvant $\mathrm{mDCF}$ to patients with nMEC in a real-world setting.

\section{Methods}

\section{Patients}

From our electronic health records, we identified all esophageal carcinoma cases histologically diagnosed and managed within our health network between August 1, 2008 and November 30, $2017(\mathrm{~N}=115)$. Only records from adult patients $(\geq 18$ years of age) who received $\mathrm{mDCF}$ for $\mathrm{nMEC}$ were considered for analysis. Imaging studies such as endoscopic ultrasound (EUS), computed tomography (CT), positron emission tomography (PET) scans, and pathology reports were further used to ascertain the stage of the esophageal tumors. Individuals who did not receive mDCF $(\mathrm{N}=74)$, had metastatic disease $(\mathrm{N}=9)$, or withdrew further treatment $(\mathrm{N}=2)$ were excluded from our final sample $(\mathrm{N}=30)$.

A retrospective chart review of the 30 cases was performed with corresponding analysis of disease-free survival (DFS), overall survival (OS), and toxicities. The analysis was conducted from the time of esophageal cancer diagnosis to time of death or disease progression/recurrence. Furthermore, we manually abstracted data on patient demographics (age and gender), tumor features (histology, grade, and stage), other concurrent treatments, hematologic toxicities, and post-treatment status. This study was approved by the Marshfield Clinic Research Institute institutional review board (IRB-18-297). 
Treatment description

All patients received an $\mathrm{mDCF}$ regimen consisting of docetaxel $40 \mathrm{mg} / \mathrm{m}^{2} \mathrm{IV}$ infusion (day 1), cisplatin $40 \mathrm{mg} / \mathrm{m}^{2} \mathrm{IV}$ (day 1), 5-fluorouracil $400 \mathrm{mg} / \mathrm{m}^{2}$ IV (day 1), 5-fluorouracil $1000 \mathrm{mg} / \mathrm{m}^{2}$ continuous IV infusion over 48 hours (days 1 and 2), and leucovorin $400 \mathrm{mg} / \mathrm{m}^{2}$ (day 1). The functional status of each patient before treatment initiation was assessed using the Eastern Cooperative Oncology Group (ECOG) performance status scale. ${ }^{23}$ The $\mathrm{mDCF}$ regimen was administered every 2 weeks with standard premedication and antiemetic treatment for a total of four to six cycles. Chemoradiation was subsequently administered with weekly doses of carboplatin and paclitaxel as radiosensitizers along with either external beam radiation or brachytherapy. Chemoradiotherapy was followed by an esophagectomy if the tumor was considered resectable. Additional medications given during the course of chemotherapy include leucovorin and granulocyte colony stimulating factors (G-CSF) such as pegfilgrastim. Hematologic toxicities were graded according to the National Cancer Institute Common Terminology Criteria for Adverse Events (version 5.0). ${ }^{24}$ Specifically, we assessed for hematological toxicities such as anemia, leukopenia, neutropenia, and thrombocytopenia.

\section{Statistical analyses}

Descriptive statistics were performed to assess patient and tumor characteristics at baseline, concurrent treatments with multimodal therapy, and hematologic toxicities. Kaplan-Meier curves were generated for analysis of DFS and OS. The DFS and OS functions were examined for each group in these variables: histology, stage, gastroesophageal junction involvement, and surgical treatment. DFS was measured from the date of diagnosis to disease recurrence/progression, whereas OS was measured from the date of diagnosis to death. Statistical analysis was performed using SAS 9.4 (SAS Institute, INC., Cary, NC, USA).

\section{Results}

Patient characteristics

Patient and tumor characteristics for our cohort are shown in Table 1. The majority of patients were men $(90 \%)$ with a median age of 64.9 years (interquartile range 61.4-72.6 years). Approximately one-third of the patients had no previous history of smoking. All patients had an ECOG performance status of $<2$ at baseline. The esophageal tumors diagnosed among these patients were mostly adenocarcinoma type (76.7\%), with esophagogastric junction (GEJ) involvement (40\%) being less frequent in the total population.

\section{Treatment and efficacy}

Table 2 describes the mDCF treatment and subsequent multimodal therapies used for these patients. All patients received radiation therapy except for two: an individual with inflammatory bowel disease (preempting radiation treatment) and another that died as a result of dehydration and cardiac complication after the fourth $\mathrm{mDCF}$ cycle. The majority of patients $(63.3 \%)$ underwent esophagectomy.
Table 1. Patient Characteristics ( $\mathrm{N}=30$ )

\begin{tabular}{|c|c|c|}
\hline Characteristic & $\mathbf{n}$ & $\%$ \\
\hline \multicolumn{3}{|l|}{ Age at diagnosis } \\
\hline \multicolumn{3}{|l|}{ Median: 64.9 years } \\
\hline \multicolumn{3}{|l|}{ IQR: $61.4-72.6$ years } \\
\hline \multicolumn{3}{|l|}{ Gender } \\
\hline Male & 27 & 90.0 \\
\hline Female & 3 & 10.0 \\
\hline \multicolumn{3}{|l|}{ Smoking } \\
\hline Current & 8 & 26.7 \\
\hline Former & 12 & 40.0 \\
\hline Never & 10 & 33.3 \\
\hline \multicolumn{3}{|l|}{ ECOG performance status } \\
\hline 0 & 28 & 93.3 \\
\hline 1 & 2 & 6.7 \\
\hline \multicolumn{3}{|l|}{ Histology } \\
\hline Squamous & 7 & 23.3 \\
\hline Adenocarcinoma & 23 & 76.7 \\
\hline \multicolumn{3}{|l|}{ Tumor site } \\
\hline Esophageal & 18 & 60.0 \\
\hline Esophagogastric junction involvement & 12 & 40.0 \\
\hline \multicolumn{3}{|l|}{ Pathology reports } \\
\hline \multicolumn{3}{|l|}{ Tumor stage } \\
\hline $\mathrm{T} 1$ & 1 & 3.3 \\
\hline T2 & 4 & 13.3 \\
\hline T3 & 23 & 76.7 \\
\hline T4 & 2 & 6.7 \\
\hline \multicolumn{3}{|l|}{ Nodal status } \\
\hline No & 12 & 40.0 \\
\hline N1 & 16 & 53.3 \\
\hline N2 & 2 & 6.7 \\
\hline \multicolumn{3}{|l|}{ Tumor size $\left(\mathrm{cm}^{2}\right)$} \\
\hline$<4$ & 11 & 36.7 \\
\hline $5-6$ & 9 & 30.0 \\
\hline $7-10$ & 8 & 26.7 \\
\hline$>10$ & 3 & 10.0 \\
\hline \multicolumn{3}{|l|}{ Grade } \\
\hline I & 1 & 3.3 \\
\hline II & 15 & 50.0 \\
\hline III & 14 & 46.7 \\
\hline \multicolumn{3}{|l|}{ Stage } \\
\hline 1 & 3 & 10.0 \\
\hline II & 14 & 46.7 \\
\hline III & 13 & 43.3 \\
\hline \multicolumn{3}{|l|}{ Other non-esophageal cancer } \\
\hline Yes & 9 & 30.0 \\
\hline No & 21 & 70.0 \\
\hline
\end{tabular}

IQR, Interquartile Range (Q1-Q3); ECOG, Eastern

Cooperative Oncology Group; mDCF, modified docetaxel, cisplatin and fluorouracil. 
Table 2. Neoadjuvant Treatment and Patient Status following mDCF Therapy $(\mathrm{N}=30)$

\begin{tabular}{lcr}
\hline Characteristic & $\mathbf{n}$ & $\%$ \\
\hline mDCF cycle & & \\
$\quad$ Median: 6 & & \\
$\quad$ IQR: 4 - 6 & & \\
Radiation & 28 & 93.3 \\
$\quad$ Yes & 2 & 6.7 \\
$\quad$ No & & \\
Surgical resection & 19 & 63.3 \\
$\quad$ Yes & 11 & 36.7 \\
$\quad$ No & & \\
Recurrence & 9 & 30.0 \\
$\quad$ Yes & 21 & 70.0 \\
$\quad$ No & & \\
Post-treatment Status & 16 & 53.3 \\
$\quad$ Alive & 14 & 46.7 \\
$\quad$ Dead & 9 & 30.0 \\
$\quad$ Recurrence & 5 & 16.7 \\
$\quad$ Not cancer related & 5 & \\
\hline mDCF, modified docetaxel, cisplatin and fluorouracil; IQR, & \\
Interquartile Range (Q1-Q3). & & \\
\hline
\end{tabular}

Figure 1 depicts the DFS and OS curves. The survival curves for DFS and OS were close to each other, with only one patient surviving with recurrent disease. Mean DFS was found to be $54.7 \pm 7.7$ months, and median OS was $56.7 \pm 7.8$ months for our cohort (squamous cell carcinoma: 76.5 months, adenocarcinoma: 44.4 months). All patients with stage I esophageal cancer were recurrence-free and alive at the end of both the second and fifth years following initial diagnosis (Table 3). Both DFS and OS decreased with increasing disease stage, histology (adenocarcinoma versus squamous), tumor location (esophageal versus GEJ involvement), and without surgical intervention. The DFS and OS mirrored each other at all-time points of follow-up in stage II, esophageal squamous cell carcinoma (SCC), GEJ involvement, and non-resected esophageal cancers, while the DFS and OS remained the same at 2-year and 5-year follow-up in esophageal SCC (71.4\%). Among the patients who did not have surgical resection (11), one underwent surgery as the study was completed; five could not have surgery due to the location of the tumor; three had adverse event(s) (two with debilitation, and one with hypotension 1), and two had no residual tumor following neoadjuvant $\mathrm{mDCF}$ and/or chemoradiotherapy.

\section{Hematologic toxicities}

As demonstrated in Table 4, Grade III or IV hematologic toxicities were less frequently seen compared to Grade I and II toxicities, with anemia and neutropenia being the least common higher-grade toxicities at $13.3 \%$ and $13.4 \%$, respectively. The number and frequency of hematological toxicities were proportional between nMEC subtypes.

\section{Discussion}

Our retrospective examination of the rates of OS, DFS, and hematologic toxicities of $\mathrm{mDCF}$ in conjunction with radiotherapy +/- surgical resection used to treat nMEC in a community setting further emphasizes the promise of this regimen's use as a neoadjuvant treatment when incorporated in multimodal therapy for nMEC, especially for patients with AC of nMEC.

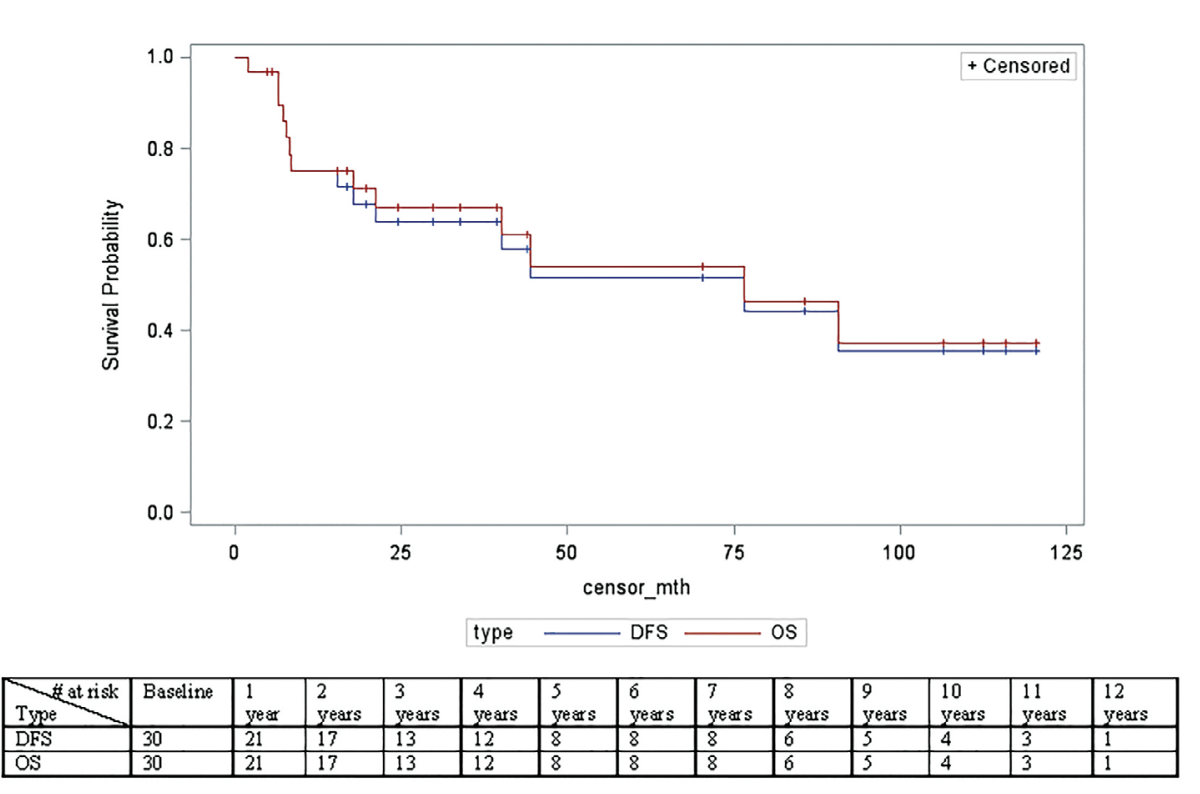

Figure 1. Comparison of DFS and OS following mDCF therapy for esophageal cancer. Note. mDCF, modified docetaxel, cisplatin, and fluorouracil; DFS, Disease-free survival; OS, Overall survival.
Examining the nMEC subpopulation of patients diagnosed with $\mathrm{AC}$ in our study, the 5-year OS was comparable to that reported by several prior studies using alternate chemotherapeutic regimens. The most frequently mentioned neoadjuvant chemotherapy is CF (cisplatin + 5-fluorouracil), while, in fact, multiple combinations and times of administration (priorand/or post-surgery) of neoadjuvant chemotherapy were proposed and studied in the last decades to identify the best treatment in patients with localized nMEC. ${ }^{25-30}$ The neoadjuvant ECX (epirubicin $50 \mathrm{mg} / \mathrm{m}^{2}$, cisplatin $60 \mathrm{mg} / \mathrm{m}^{2}$, and capecitabine $1250 \mathrm{mg} /$ $\mathrm{m}^{2}$; median survival: 26.1 months) and neoadjuvant CF (cisplatin $80 \mathrm{mg} / \mathrm{m}^{2}$ and fluorouracil $1000 \mathrm{mg} / \mathrm{m}^{2}$; median survival: 23.4 months) were compared in the OE05 trial conducted in England. ${ }^{29}$ Patients with surgically 
Table 3. Disease-Free and Overall Survival following mDCF Therapy According to Tumor Features and Surgical Treatment $(\mathrm{N}=30)$

\begin{tabular}{|c|c|c|c|c|c|c|}
\hline \multirow[b]{2}{*}{ Variable } & \multicolumn{3}{|c|}{ Disease-Free Survival } & \multicolumn{3}{|c|}{ Overall Survival } \\
\hline & 2-year, \% & 3-year, \% & 5-year, \% & 2-year, \% & 3-year, \% & 5 -year, \% \\
\hline \multicolumn{7}{|l|}{ Stage } \\
\hline 1 & 100.0 & 100.0 & 100.0 & 100.0 & 100.0 & 100.0 \\
\hline II & 68.8 & 68.8 & 51.6 & 68.8 & 68.8 & 51.6 \\
\hline III & 50.0 & 50.0 & 37.5 & 57.1 & 57.1 & 42.9 \\
\hline \multicolumn{7}{|l|}{ Histology } \\
\hline Adenocarcinoma & 60.8 & 60.8 & 41.7 & 64.9 & 64.9 & 44.5 \\
\hline Squamous & 71.4 & 71.4 & 71.4 & 71.4 & 71.4 & 71.4 \\
\hline \multicolumn{7}{|l|}{ Location } \\
\hline Esophageal & 66.7 & 66.7 & 57.1 & 72.2 & 72.2 & 61.9 \\
\hline GEJ involvement & 60.0 & 60.0 & 45.0 & 60.0 & 60.0 & 45.0 \\
\hline \multicolumn{7}{|l|}{ Surgical resection } \\
\hline Yes & 65.3 & 65.3 & 54.4 & 69.9 & 69.9 & 58.3 \\
\hline No & 60.6 & 60.6 & 48.5 & 60.6 & 60.6 & 48.5 \\
\hline Overall & 63.9 & 63.9 & 51.6 & 67.0 & 67.0 & 54.2 \\
\hline
\end{tabular}

GEJ, Gastroesophageal junction; mDCF, modified docetaxel, cisplatin, and fluorouracil.

resectable nMEC AC had a better median survival with neoadjuvant ECX (26.1 months) than those with neoadjuvant CF (23.4 months)..$^{29}$ However, Grade III/IV neutropenia rate was higher with ECX $(23 \%)$ than CF $(17 \%){ }^{29}$ Our study, on the other hand, shows a promising profile in both safety and survival outcome: $13 \%$ Grade III/IV neutropenia and 44 months median survival time in the nMEC AC subgroup. A similar median survival time in the CROSS trial has been observed in neoadjuvant chemoradiotherapy comprising taxane + platinum and radiation (41.4 Gy). ${ }^{28,30}$ In the CROSS trial, patients with resectable nMEC were treated either with surgery only or neoadjuvant chemoradiotherapy followed by surgery; the neoadjuvant chemoradiotherapy comprised five cycles of neoadjuvant PC (paclitaxel $50 \mathrm{mg} / \mathrm{m}^{2}$, carboplatin AUC $2 \mathrm{mg} /$ $\mathrm{mL}$ per min on day $1,8,15,22$ and 29) concurrent with radiotherapy (23 fractions of 1.8 Gy starting on the first day of the first chemotherapy, 5 days per week in weeks 1, 2, 3, and 4, then 3 days in week 5). ${ }^{28,30}$ Findings in the CROSS trial showed patients treated with neoadjuvant chemoradiotherapy demonstrated better survival outcomes (median survival time: 43.2 vs. 24.0 months) compared to those treated with surgery only, and neoadjuvant chemoradiotherapy (taxane + platinum + radiation) was associated with less severe adverse events (Grade III/IV neutropenia: 2\%, Grade III/IV leukopenia: 6\%, Grade III/

Table 4. Hematologic Toxicities During mDCF Treatment $(\mathrm{N}=30)$

\begin{tabular}{lrrrrc}
\hline & \multicolumn{5}{c}{ Grade $^{\mathrm{a}}$} \\
\cline { 2 - 5 } Toxicity Type & $\mathbf{1}$ & $\mathbf{2}$ & $\mathbf{3}$ & $\mathbf{4}$ & $\mathbf{3} / 4$ \\
$\mathbf{N}(\%)$ & $\mathbf{N}(\%)$ & $\mathbf{N}(\%)$ & $\mathbf{N}(\%)$ & $\mathbf{N}(\%)$ \\
\hline Anemia & $15(50.0)$ & $11(36.7)$ & $4(13.3)$ & $0(0.0)$ & $4(13.3)$ \\
$\quad$ Squamous cell carcinoma (N=7) & $5(71.4)$ & $2(28.6)$ & $0(0.0)$ & $0(0.0)$ & \\
$\quad$ Adenocarcinoma (N=23) & $10(43.5)$ & $9(39.1)$ & $4(17.4)$ & $0(0.0)$ & \\
Leukopenia & $14(46.7)$ & $3(10.0)$ & $11(36.7)$ & $2(6.7)$ & $13(43.4)$ \\
$\quad$ Squamous cell carcinoma (N=7) & $4(57.1)$ & $0(0.0)$ & $2(28.6)$ & $1(14.3)$ & \\
$\quad$ Adenocarcinoma (N=23) & $10(43.5)$ & $3(13.0)$ & $9(39.1)$ & $1(4.4)$ & \\
Neutropenia & $15(50.0)$ & $11(36.7)$ & $2(6.7)$ & $2(6.7)$ & $4(13.3)$ \\
$\quad$ Squamous cell carcinoma (N=7) & $4(57.1)$ & $2(28.6)$ & $0(0.0)$ & $1(14.3)$ & \\
$\quad$ Adenocarcinoma (N=23) & $11(47.8)$ & $9(39.1)$ & $2(8.7)$ & $1(4.4)$ & \\
Thrombocytopenia & $19(63.3)$ & $6(20.9)$ & $5(16.7)$ & $0(0.0)$ & $5(16.7)$ \\
$\quad$ Squamous cell carcinoma $(\mathrm{N}=7)$ & $5(71.4)$ & $2(28.8)$ & $0(0.0)$ & $0(0.0)$ & \\
$\quad$ Adenocarcinoma (N=23) & $14(60.9)$ & $4(17.4)$ & $5(21.7)$ & $0(0.0)$ & \\
\hline
\end{tabular}

${ }^{a}$ Grading was defined using the National Cancer Institute Common Terminology Criteria for Adverse Events version 5.0. (Finnigan 2018) 
IV thrombocytopenia: 1\%) than neoadjuvant mDCF (taxane + platinum $+5-\mathrm{FU}$ ) in our study (neutropenia: $13 \%$, leukopenia: $43 \%$, thrombocytopenia: $17 \%$ ). ${ }^{30}$ With all evidence from these different studies, we summarized that the combination of taxane and platinum might be a better mainstay of neoadjuvant for nMEC AC than CF (cisplatin and platinum), because less severe adverse events were observed. However, different compounds under the same category might have different safety profiles, and so further studies focused on neoadjuvant chemotherapy with taxane and platinum are encouraged.

Studies have shown the effectiveness of $\mathrm{mDCF}$ in patients with nMEC AC, while providers need to pay more attention to the dosage of $\mathrm{mDCF}$, interval among cycle, and administration time. A multicenter phase II trial of perioperative DCF combined with surgical resection for advanced nMEC patients reported a 3 -year OS of $60 \%$ with $14-22 \%$ Grade III/IV neutropenia. ${ }^{15}$ In our study, not only did nMEC AC patients treated with neoadjuvant $\mathrm{mDCF}$ have good survival outcomes (3-year and 5-year OS of $64.9 \%$ and $44.5 \%$ ), but patients also suffered less from severe neutropenia (13\%). However, one should be aware of the differences that exist between the two studies, making it hard to perform a comparative effectiveness study. For example, all patients in Ferri's study had an advanced nMEC diagnosis and were treated with perioperative mDCF + surgery. Only $63 \%$ of our patients had surgery after the four to six cycle adjuvant mDCF. ${ }^{15}$ Examined closely, the dosages of modified DCF and interval of the cycle were different between the two studies. Three 3-week cycles mDCF (docetaxel $75 \mathrm{mg} / \mathrm{m}^{2}$ on day 1 , cisplatin $75 \mathrm{mg} / \mathrm{m}^{2}$ on day 1,5 -FU $750 \mathrm{mg} / \mathrm{m}^{2}$ for 120 hours) were provided before and after surgery in Ferri's, while four 6-week mDCF (docetaxel $40 \mathrm{mg} / \mathrm{m}^{2}$ on day 1 , cisplatin 40 $\mathrm{mg} / \mathrm{m}^{2}$ on day $1,5-\mathrm{FU} 400 \mathrm{mg} / \mathrm{m}^{2}$ for 48 hours, leucovorin $400 \mathrm{mg} / \mathrm{m}^{2}$ on day 1) were administrated before chemoradiotherapy + surgery. The dosage of individual compounds and interval between cycles play an essential role in the effectiveness and toxicity of $\mathrm{mDCF}$ as well. Simply put, both studies have shown that $\mathrm{mDCF}$ has a greater potential for better outcomes in patients with local and localadvanced nMEC AC, but further studies with more rigid study design and head-to-head comparisons to address confounding factors are needed.

The mDCF regimen also demonstrates promising results for locally advanced esophageal SCC compared to the parent DCF regimen. In a 2012 study by Katada and colleagues, ${ }^{31}$ the preioperative administration of DCF demonstrated better OS and DFS compared to CF for the treatment of locally advanced, resectable esophageal SCC. The DCF regimen in Katada's study included docetaxel 70-75 $\mathrm{mg} / \mathrm{m}^{2}$ (day 1), cisplatin $70-75 \mathrm{mg} / \mathrm{m}^{2}$ (day 1), and 5-fluorouracil $750 \mathrm{mg} / \mathrm{m}^{2}$ IV infusion (day 1-5) and was repeated twice every 3 weeks. The 2-year OS and DFS of study subjects were $93 \%$ and $68 \%$, respectively. The Grade III/IV hematological toxicities were a major concern, with $87 \%$ of patients experiencing leukopenia, and 92\% experiencing neutropenia. ${ }^{31}$ The 2-year OS and DFS of our mDCF regimen was $71.4 \%$ with lower rates of Grade III/IV hematological toxicity (43\% leukopenia and $14 \%$ neutropenia). Higher dosages of DCF seem to correlate with better survival outcomes, yet the high rates of serious adverse events are often negatively associated with disease-related quality of life. While a direct comparison to patients in the Katada study ${ }^{31}$ is difficult, especially in light of racial/ethnic differences (Japanese vs. Americans), sample size, and the addition of chemoradiation in our study, the relatively favorable DFS and OS of the $\mathrm{mDCF}$ regimen with much lower rates of toxicity suggests that minor adjustments in DCF dosages may be a promising treatment option for patients with locally advanced SCC of the esophagus. Again, further studies are needed for direct comparisons in this SCC subpopulation.

A few limitations to our study should be addressed. First, as this was a retrospective study, we were limited by factors inherent to this study design, such as less strict inclusion and exclusion criteria and certain essential information missing in the charts. Data were collected for routine clinical care rather than for research purposes, and no control group was used for comparison. When we reviewed and obtained patient information for analysis (such as DFS), we might not have captured all disease-related information if not explicitly documented in the EHR, resulting in slight differences in DFS and OS. Lastly, our study population was relatively small and homogeneous concerning race/ethnicity (approximately 90\% non-Hispanic Whites vs. $77 \%$ of the general US population), which limits the generalizability of our results.

\section{Conclusion}

Our results suggest that $\mathrm{mDCF}$ as part of a multimodal treatment regimen for nMEC is an effective and tolerable neoadjuvant chemotherapy regimen used in a community setting. Further studies are needed for direct comparisons.

\section{Acknowledgements}

The authors acknowledge Emily A. Andreae, PhD and Marie Fleisner from Marshfield Clinic Research Institute for assistance with manuscript editing and submission; and Ben George, MD from Medical College of Wisconsin for his scientific critique.

\section{References}

1. Siegel RL, Miller KD, Jemal A. Cancer statistics, 2019. CA Cancer J Clin. 2019;69(1):7-34.

2. Cronin KA, Lake AJ, Scott S, et al. Annual Report to the Nation on the Status of Cancer, part I: National cancer statistics. Cancer. 2018;124(13):2785-2800.

3. Howlader N, Noone A, Krapcho M, et al. SEER Cancer Statistics Review. 1975-2016 Bethesda, MD: National Cancer Institutes; 2018. Available at https://seer.cancer. gov/csr/1975_2016/ 
4. Noone A, Howlader N, Krapcho M, et al. Cancer Facts \& Figures 2019. Atlanta: American Cancer Society; 2019. Available at https://www.cancer.org/content/dam/ cancer-org/research/cancer-facts-and-statistics/annual-cancer-facts-and-figures/2019/cancer-facts-and-figures-2019.pdf. Last accessed March 25, 2021.

5. Nassri A, Zhu H, Muftah M, Ramzan Z. Epidemiology and survival of esophageal cancer patients in an American cohort. Cureus. 2018;10(4):e2507.

6. Kauppila JH, Mattsson F, Brusselaers N, Lagergren J. Prognosis of oesophageal adenocarcinoma and squamous cell carcinoma following surgery and no surgery in a nationwide Swedish cohort study. BMJ Open. 2018;8(5):e021495.

7. Pennathur A, Luketich JD. Resection for esophageal cancer: strategies for optimal management. Ann Thorac Surg. 2008;85(2):S751-S756.

8. Rüdiger Siewert J, Feith M, Werner M, Stein HJ. Adenocarcinoma of the esophagogastric junction: results of surgical therapy based on anatomical/topographic classification in 1,002 consecutive patients.

Ann Surg. 2000;232(3):353-361.

9. Sohda M, Kuwano H. Current status and future prospects for esophageal cancer treatment. Ann Thorac Cardiovasc Surg. 2017;23(1):1-11.

10. Ajani JA, D’Amico TA, Almhanna K, et al; National comprehensive cancer network. Esophageal and esophagogastric junction cancers, version 1.2015. J Natl Compr Canc Netw. 2015;13(2):194-227.

11. Lordick F, Mariette C, Haustermans K, Obermannová R, Arnold D; ESMO Guidelines Committee. Oesophageal cancer: ESMO Clinical Practice Guidelines for diagnosis, treatment and follow-up. Ann Oncol. 2016;27(suppl 5):v50-v57.

12. Sjoquist KM, Burmeister BH, Smithers BM, et al; Australasian Gastro-Intestinal Trials Group. Survival after neoadjuvant chemotherapy or chemoradiotherapy for resectable oesophageal carcinoma: an updated meta-analysis. Lancet Oncol. 2011;12(7):681-692.

13. Chan KKW, Saluja R, Delos Santos K, et al. Neoadjuvant treatments for locally advanced, resectable esophageal cancer: A network meta-analysis. Int J Cancer. 2018;143(2):430-437.

14. Ashok A, Tiwari V, Jiwnani S, Karimundackal G, Pramesh CS. Controversies in preoperative therapy in esophageal cancer: Current evidence and ongoing research. Annals of Gastroenterological Surgery. 2019;3(6):592-597.

15. Ferri LE, Ades S, Alcindor T, et al. Perioperative docetaxel, cisplatin, and 5-fluorouracil (DCF) for locally advanced esophageal and gastric adenocarcinoma: a multicenter phase II trial. Ann Oncol. 2012;23(6):1512-1517.
16. Van Cutsem E, Moiseyenko VM, Tjulandin S, et al; V325 Study Group. Phase III study of docetaxel and cisplatin plus fluorouracil compared with cisplatin and fluorouracil as first-line therapy for advanced gastric cancer: a report of the V325 Study Group. J Clin Oncol. 2006;24(31):49914997.

17. Miyazaki T, Sohda M, Tanaka N, et al. Phase I/II study of docetaxel, cisplatin, and 5-fluorouracil combination chemoradiotherapy in patients with advanced esophageal cancer. Cancer Chemother Pharmacol. 2015;75(3): 449-455.

18. Miyawaki Y, Nakajima Y, Kawada K, Okada T, Tokairin Y, Kawano T. Efficacy of docetaxel, cisplatin, and 5-fluorouracil chemotherapy for superficial esophageal squamous cell carcinoma. Dis Esophagus. 2017;30:1-8.

19. Pasini F, de Manzoni G, Pedrazzani C, et al. High pathological response rate in locally advanced esophageal cancer after neoadjuvant combined modality therapy: dose finding of a weekly chemotherapy schedule with protracted venous infusion of 5-fluorouracil and dose escalation of cisplatin, docetaxel and concurrent radiotherapy. Ann Oncol. 2005;16(7):1133-1139.

20. Pasini F, de Manzoni G, Zanoni A, et al. Neoadjuvant therapy with weekly docetaxel and cisplatin, 5-fluorouracil continuous infusion, and concurrent radiotherapy in patients with locally advanced esophageal cancer produced a high percentage of long-lasting pathological complete response: a phase 2 study. Cancer. 2013;119(5):939-945.

21. Shah MA, Jhawer M, Ilson DH, et al. Phase II study of modified docetaxel, cisplatin, and fluorouracil with bevacizumab in patients with metastatic gastroesophageal adenocarcinoma. J Clin Oncol. 2011;29:868-874.

22. Shah MA, Janjigian YY, Stoller R, et al. Randomized multicenter phase II study of modified docetaxel, cisplatin, and fluorouracil (DCF) versus DCF plus growth factor support in patients with metastatic gastric adenocarcinoma: a study of the US gastric cancer consortium. J Clin Oncol. 2015;33:3874-3879.

23. Oken M, Creech R, Tormey D, et al. Toxicity and response criteria of the Eastern Cooperative Oncology Group. Am J Clin Oncol. 1982;5:649-655.

24. Finnigan S. Common Terminology Criteria for Adverse Events (CTCAE) Bethesda, MD; 2018. Available at https://ctep.cancer.gov/protocolDevelopment/electronic_ applications/ctc.htm\#ctc_50. Last accessed: March 25, 2021.

25. Allum WH, Stenning SP, Bancewicz J, Clark PI, Langley RE. Long-term results of a randomized trial of surgery with or without preoperative chemotherapy in esophageal cancer. J Clin Oncol. 2009;27:5062-5067. 
26. Kelsen DP, Ginsberg R, Pajak TF, et al. Chemotherapy followed by surgery compared with surgery alone for localized esophageal cancer. N Engl J Med. 1998;339:1979-1984.

27. Kelsen DP, Winter KA, Gunderson LL, et al. Long-term results of RTOG trial 8911 (USA Intergroup 113): a random assignment trial comparison of chemotherapy followed by surgery compared with surgery alone for esophageal cancer. J Clin Oncol. 2007;25:3719-3725.

28. Shapiro J, Lanschot JJB van, Hulshof MCCM, et al. Neoadjuvant chemoradiotherapy plus surgery versus surgery alone for oesophageal or junctional cancer (CROSS): long-term results of a randomised controlled trial. Lancet. 2015;16:1090-1098.

29. Alderson D, Cunningham D, Nankivell M, et al. Neoadjuvant cisplatin and fluorouracil versus epirubicin, cisplatin, and capecitabine followed by resection in patients with oesophageal adenocarcinoma (UK MRC OE05): an open-label, randomised phase 3 trial. Lancet Oncol. 2017;18:1249-1260.

30. van Hagen P, Hulshof MCCM, van Lanschot JJB, et al. Preoperative Chemoradiotherapy for Esophageal or Junctional Cancer. N Engl J Med. 2012;366:2074-2084.

31. Katada N, Yamashita K, Katada C, et al. Neoadjuvant chemotherapy using concurrent Docetaxel/CDDP/5-FU (DCF) in esophageal squamous cell carcinoma and its short-term prognosis. Esophagus. 2014;11:173-181.

\section{Author Affiliations}

Adedayo A. Onitilo, MD, PhD, MSCR, FACP ${ }^{*}$, ; Trista J. Stankowski-Drengler, MD, MS; Oyewale Shiyanbola, MBBS, MPH, PhD\$; Jessica Engel, DNP, MS*; Sabo Tanimu, $M D^{\prime}$; Seth O. Fagbemi, MD*; and Ya-Huei Li, PhD"

*Department of Hematology/Oncology, Marshfield ClinicWeston Center, Weston, Wisconsin Cancer Care and Research Center, Marshfield Clinic

Research Institute, Marshfield, Wisconsin

* Wisconsin Surgical Outcomes Research Program, Department of Surgery, University of Wisconsin School of Medicine and Public Health, Madison, Wisconsin

${ }^{\S}$ Department of Pathology and Laboratory Medicine, University of Wisconsin School of Medicine and Public Health, Madison, Wisconsin

'Department of Gastroenterology, Marshfield Clinic-Weston Center, Weston, Wisconsin 